\title{
Preface to The Relativistic Quantum Chemistry Issue of Journal of Computer Chemistry, Japan
}

\author{
Pekka PYYKKÖa \\ aDepartment of Chemistry, University of Helsinki, POB 55 (A. I. Virtasen aukio 1), 00014 Helsinki, Finland \\ *e-mail: pekka.pyykko@helsinki.fi \\ A concise review of the landscape of Relativistic Quantum Chemistry is given, and related to the papers in the \\ present issue.
}

Keywords: Relativistic Quantum Chemistry, Heavy-element compounds.

Relativistic Quantum Chemistry (RQC) has become a large and fertile field. The current version 17.1. of the RTAM database (rtam.csc.fi) [1] has 16678 entries. Of course, Nature is relativistic, and only relativistic, but the previous models, before RQC, were non-relativistic and it is interesting to know, how large the changes are, when the relativistic effects are introduced. A brief answer [2] is that many of the differences from the 5th Period to the 6th Period then receive a rational explanation. In a way we are then crossing the special relativity of Einstein with the Periodic System of Mendeleev (and others). The valence-shell relativistic effects, down a column, roughly grow as $Z^{2}$, the square of the full nuclear charge, $Z$. In very high-precision work one already needs RQC and Quantum Electrodynamics (QED) effects for hydrogen, $Z=1$.

Indeed, our first question is to ask, how complete is our physical model? Taking the Dirac-Fock-Breit (DFB) Hamiltonian as the starting point, the largest missing piece of Physics seems to be the Lamb shift. To lowest order it has the Self-Energy (SE) and Vacuum Polarization (VP) terms, of opposite sign and in this order of importance. For the ns valence electrons of heavier Group 11 elements, they together are about $-1 \%$ of the Dirac-level relativistic effects. In that sense, the DFB seems to be "101\% right." For a review on the 'Physics behind Chemistry', see the recent paper [3]. The next physical terms, take parity non-conservation, are many powers of ten further down [4]. Should one use the hypothetical reference of a point nucleus, the finite-nucleus effects become comparable to QED for large $Z$, see [3], Figure 3.

Assuming, then, that we have a reasonably firm physical basis, how well can we do the actual calculation? Sometimes the calculation becomes easier, if a transformation of the original DFB Hamiltonian is used. An example is the Douglas-KrollHess (DKH) approximation, to some order. The first paper by Nakajima [5] is a review on this example. The DKH-like approaches can be taken to infinite order, see the review by Nakai and Seino [6].

The next question is the way of handling electron correlation. If the Hartree-Fock level is taken as the starting point, one sequence is the Møller-Plesset MPn series. Another sequence, obtained by summing single, double, etc. virtual excitations to infinite order, is the coupled-cluster sequence CCSD, CCSD (T) etc. The reference does not have to be a single configuration. Multireference Perturbation Theories are discussed here by Suzuki et al. [7]. A shortcut to get the electron-electron cusp right is to introduce the interelectronic coordinates to the wavefunction. These are the so-called $R_{12}$ (or $F_{12}$ ) methods. Finally, a Gordian solution to the correlation problem is the Density Functional Theory, DFT. It is a very economic approach and makes even large molecules tractable. DFT is seldom very wrong. In this volume the intersection of DFT and relativistic calculations is treated by Tsuneda [8].

A further axis is that of the basis sets. Traditionally, think- 
ing of Slater basis functions, approximated by Gaussians, one speaks of single-, double-, triple- etc. $\zeta$ basis, where $\zeta$ is the effective Slater exponent. If relativistic pseudopotentials are developed, the appropriate basis sets usually come with them.

One particularity of relativistic wavefunctions is the spinorbit (SO) splitting of non- $s$ atomic states. For instance, the atomic $p$ levels are split into $p_{1 / 2}$ and $p_{3 / 2}$ levels. For molecules, this necessitates the use of double groups for the electronic states. This is the case as soon as the lowest-order SO is used. An SO configuration interaction method by Yabushita [9] is included here.

"The proof of the pudding is in the eating." A very concrete example is isotope separation. For the heavier elements the isotope shifts are driven by nuclear-volume effects. Two particular separations are the uranium isotopes U-235 and U-238, or the isotopes of gadolinium, discussed in the present volume by Abe et al. [10]. This slight difference of equilibrium constants has an importance both in industrial-level processes and in geochemistry or cosmochemistry. Another application of RQC is the hydration of divalent alkaline earth ions, discussed here by Mori and Matsuda [11].

A third, refreshingly different yet real, application is that on positronic compounds by Tachikawa and Kita [12]. Because the net charge of the nucleus + electrons is zero for a neutral molecule, the positron is presumably bound by the combined multipole moments of that molecule. Forgetting the higher multipoles, as well as induction, dispersion etc., we can start with the dipole moment. Such a correlation between the positron binding energy and the molecular dipole moment was found here by Tachikawa and Kita [12]. Although the positron never moves fast, the mentioned dipole moment can be strongly influenced by relativity.

For the record, the problem of an electron (or positron) in the field of a point dipole goes back to Fermi and Teller [13]. For the Schrödinger equation, the present dipole moment binding limit is 0.639314877199981 au [14]. The electron binding to higher multipoles has also been studied, see e.g [15].

Finally, there is room for interpretative considerations of the Dirac Equation [16].

\section{References}

[1] P. Pyykkö, J. Comput. Chem., 34, 2667 (2013). [CrossRef] [Medline]

[2] P. Pyykkö, Adv. Quantum Chem., 11, 353 (1978).[CrossRef]

[3] P. Pyykkö, Chem. Rev., 112, 371 (2012). [Medline] [CrossRef]

[4] P. Pyykkö, (2012), See ref. 26 of ref. [3].

[5] T. Nakajima, J. Comput. Chem. Jpn., 13, 50 (2014).

[6] H. Nakai, J. Seino, J. Comput. Chem. Jpn., 13, 1 (2014).

[7] S. Suzuki, R. Ebisuzaki, Y. Watanabe, H. Nakano, J. Comput. Chem. Jpn., 13, 32 (2014).

[8] T. Tsuneda, J. Comput. Chem. Jpn., 13, 71 (2014).

[9] S. Yabushita, J. Comput. Chem. Jpn., 13, 43 (2014).

[10] M. Abe, M. Hada, T. Suzuki, Y. Fujii, K. Hirao, J. Comput. Chem. Jpn., 13, 92 (2014).

[11] H. Mori, A. Matsuda, J. Comput. Chem. Jpn., 13, 105 (2014).

[12] M. Tachikawa, Y. Kita, J. Comput. Chem. Jpn., 13, 83 (2014).

[13] E. Fermi, E. Teller, Phys. Rev., 72, 399 (1947). [CrossRef]

[14] A. D. Alhaidari, H. Bahlouli, Phys. Rev. Lett., 100, 110401 (2008). [Medline] [CrossRef]

[15] V. I. Pupyshev, A. Y. Ermilov, Int. J. Quantum Chem., 96, 185 (2004). [CrossRef]

[16] A. Tachibana, J. Comput. Chem. Jpn., 13, 18 (2014). 